\title{
Longitudinal optical imaging of tumor metabolism and hemodynamics
}

\author{
Melissa C. Skala \\ Andrew Fontanella \\ Duke University \\ Department of Biomedical Engineering \\ 136 Hudson Hall, Box 90281 \\ Durham, North Carolina 27708
}

Lan Lan

Duke University

Department of Biostatistics and Bioinformatics

Box 2721

Durham, North Carolina 27710

\author{
Joseph A. Izatt \\ Duke University \\ Department of Biomedical Engineering \\ 136 Hudson Hall, Box 90281 \\ Durham, North Carolina 27708
}

Mark W. Dewhirst

Duke University

Department of Radiation Oncology

Box 3455

Durham, North Carolina 27710

\begin{abstract}
An important feature of tumor hypoxia is its temporal instability, or "cycling hypoxia." The primary consequence of cycling hypoxia is increased tumor aggressiveness and treatment resistance beyond that of chronic hypoxia. Longitudinal imaging of tumor metabolic demand, hemoglobin oxygen saturation, and blood flow would provide valuable insight into the mechanisms and distribution of cycling hypoxia in tumors. Fluorescence imaging of metabolic demand via the optical redox ratio (fluorescence intensity of FAD/ $\mathrm{NADH}$ ), absorption microscopy of hemoglobin oxygen saturation, and Doppler optical coherence tomography of vessel morphology and blood flow are combined to noninvasively monitor changes in oxygen supply and demand in the mouse dorsal skin fold window chamber tumor model (human squamous cell carcinoma) every $6 \mathrm{~h}$ for $36 \mathrm{~h}$. Biomarkers for metabolic demand, blood oxygenation, and blood flow are all found to significantly change with time $(p<0.05)$. These variations in oxygen supply and demand are superimposed on a significant $(p<0.05)$ decline in metabolic demand with distance from the nearest vessel in tumors (this gradient was not observed in normal tissues). Significant $(p<0.05)$, but weak $(r \leqslant 0.5)$ correlations are found between the hemoglobin oxygen saturation, blood flow, and redox ratio. These results indicate that cycling hypoxia depends on both oxygen supply and demand, and that noninvasive optical imaging could be a valuable tool to study therapeutic strategies to mitigate cycling hypoxia, thus increasing the effectiveness of radiation and chemotherapy. () 2010 Society of Photo-Optical Instrumentation Engineers. [DOI: 10.1117/1.3285584]
\end{abstract}

Keywords: tumor hypoxia; cycling; longitudinal imaging; tumor metabolism.

Paper 09287SSR received Jul. 7, 2009; revised manuscript received Oct. 26, 2009; accepted for publication Oct. 27, 2009; published online Jan. 13, 2010.

\section{Introduction}

Hypoxic tumors are more aggressive than their normoxic counterparts, and are resistant to radiation and chemotherapy. ${ }^{1-3}$ Thus, a major goal of cancer research is to gain a better understanding of oxygen supply and demand in tumors. Studies of oxygen supply and demand could provide a basis for improved prognostic and treatment approaches for solid tumors. An important feature of tumor hypoxia is its temporal instability. "Cycling hypoxia" has been described as a pattern of temporal periodicity between hypoxic and reoxygenated states. It has been observed in many tumor types, ${ }^{4-6}$ whereas studies measuring $\mathrm{pO}_{2}$ fluctuations in normal tissue (muscle) in rats and mice have not observed significant fluctuations. ${ }^{7,8}$ One primary consequence of cycling hypoxia is upregulation of the transcription factor hypoxia-inducible factor 1 (HIF1) activity to a level that supersedes that of chronic hypoxia. This transcription factor controls many cel-

Address all correspondence to Melissa C. Skala, Duke University, Department of Biomedical Engineering, 136 Hudson Hall, Box 90281, Durham, North Carolina 27708. Tel: 919-660-5588; Fax: 919-684-4488; E-mail: melissa.skala@duke.edu lular functions that exacerbate treatment resistance and tumor aggressiveness. ${ }^{9}$

Cycling hypoxia could be caused by changes in tumor vascular function (oxygen delivery) and metabolic demand. In tumors, formation of new vessels and remodeling and dropout of existing vessels occurs continuously, resulting in temporal variations in blood flow and blood oxygenation. ${ }^{10}$ Oxygen consumption rate is a highly dynamic feature of oxygen transport in tumors, because small changes in demand for oxygen create large changes in the extent and severity of hypoxia. ${ }^{11}$ Longitudinal imaging of tumor metabolic demand, vessel morphology, hemoglobin oxygen saturation, and blood flow velocities would provide valuable insight into the mechanisms and distribution of cycling hypoxia in tumors. For example, these data will allow for the study of tumor response to changing hemodynamic and metabolic conditions induced by experimental and traditional cancer therapies. Such information can also be used to test and refine theoretical models for structural adaptation in tumors, which have successfully predicted tumor response to drug and radiation therapy. ${ }^{12}$

1083-3668/2010/15(1)/011112/8/\$25.00 @ 2010 SPIE 
Current methods for measuring oxygen supply on the microvessel level include microelectrode measurements, ${ }^{7}$ and phosphorescence lifetime imaging of a $\mathrm{pO}_{2}$ calibrated dye. ${ }^{13}$ Microelectrodes provide a sensitive and direct measure of $p \mathrm{O}_{2}$, but are inherently invasive and limited to point measurements. Phosphorescence lifetime imaging provides information on the 2-D distribution of oxygen within the tumor, but requires the injection of a contrast agent. The use of a contrast agent is invasive and may require repeated exposure to the dye for longitudinal measurements, which further complicates data acquisition and interpretation. Blood flow on the microvessel level has traditionally been measured using laser Doppler flowmetry, ${ }^{7}$ the dual-slit method, ${ }^{13}$ or video microscopy. ${ }^{14,15}$ Laser Doppler flowmetry provides a relative (not calibrated) measurement of blood flow at a single point, and scanning laser Doppler provides an average flow over a tissue area without accounting for contributions from individual vessels. The dual-slit technique and video microscopy both suffer from inaccurate estimations of the length traveled by red blood cells because the length of a 3-D vessel is projected onto a 2-D image, resulting in imprecise velocity values. Video microscopy of fluorescently labeled red blood cell flux and blood flow rate can be done, but is only practical for vessels less than $\sim 30 \mu \mathrm{m}$ in diameter because individual red blood cells are difficult to visualize in larger vessels due to absorption of fluorescent light by hemoglobin.

Previous methods for measuring oxygen demand in tumors include $p \mathrm{O}_{2}$ microelectrode measurements coupled with theoretical simulations of oxygen diffusion, ${ }^{16}$ an isolated tumor perfusion system combined with Fick's principle calculations, ${ }^{17}$ and cryospectrophotometric microtechniques. ${ }^{18}$ All of these methods are invasive, which makes longitudinal in vivo monitoring difficult. Positron emission tomography (PET) of fluoro-deoxyglucose (FDG) has also served as a valuable marker for tumor metabolism. ${ }^{19,20}$ However, PET imaging is low resolution and expensive, and time course measurements require repeated exposure to radioisotopes.

Optical imaging of tumor oxygen supply and demand is attractive because these methods are relatively low cost, high resolution, and noninvasive, if performed in a transparent window chamber preparation. Optical imaging based on endogenous tissue contrast does not require exogenous contrast agents, and is thus well suited for long-term monitoring of cycling hypoxia in tumors. Hyperspectral imaging of hemoglobin oxygen saturation ${ }^{21-23}$ coupled with 3-D Doppler optical coherence tomography (OCT) of calibrated microvessel blood flow rates ${ }^{24-27}$ allow for accurate, dynamic, and noninvasive imaging of oxygen supply in tumors. ${ }^{28}$ Doppler OCT also allows for visualization of vessel velocity profiles at any vessel cross section in the tumor, thus providing information on the shear rate on the vessel wall. ${ }^{29}$ Oxygen demand can be optically monitored with the "redox ratio," which is the ratio of the inherent fluorescence intensity of the metabolic coenzymes FAD and NADH (the primary electron acceptor and donor, respectively, in oxidative phosphorylation.). ${ }^{30}$ This optical redox ratio provides relative changes in the oxidationreduction state in the cell. The redox ratio is sensitive to changes in the cellular metabolic rate and vascular oxygen supply. ${ }^{30-34}$
The goal of this study was to dynamically image changes in oxygen supply and demand in tumors using a novel combination of redox imaging (metabolic demand) and hyperspectral imaging, combined with Doppler OCT (oxygen supply). The results reported here are the first demonstration of combined noninvasive imaging of oxygen supply and metabolic demand in tumors in vivo. These data could provide insight into the sources of cycling hypoxia in tumors, and serve as the basis for further studies that will incorporate therapeutic strategies to mitigate hypoxia in tumors for improved treatment response.

\section{Materials and Methods}

\subsection{Multifunctional Microscope}

Fluorescence, hyperspectral, and OCT imaging were conducted on the same microscope to noninvasively monitor changes in metabolic demand and hemodynamics with tumor growth. All images were collected with a $4 \times$ objective (NA $=0.1$ ). Hyperspectral (hemoglobin oxygen saturation) and SDOCT (blood flow and vascular morphology) arms were connected through two separate base ports in an inverted microscope (Carl Zeiss Axiovert 200). 2-D hyperspectral images were collected with a $100-\mathrm{W}$ halogen lamp for transillumination, and detection was achieved with a 10-nm bandwidth liquid crystal tunable filter (LCTF) (CRI Incorporated, Woburn, Massachusetts) placed in front of a DVC 1412 CCD camera (DVC Company, Austria, Texas). ${ }^{23}$ Custom software was used to tune the filter and acquire images at $10-\mathrm{nm}$ increments between 500 and $620 \mathrm{~nm}$. Measurements of the dark offset and transmission through a neutral density filter at each wavelength were made before each imaging session. Hemoglobin saturation images were calculated by applying an extension of the Beer-Lambert law to the wavelength-dependent attenuation at each pixel (assuming oxygenated and deoxygenated hemoglobin as the primary absorbers, along with a tissue scattering parameter), and then solving for hemoglobin saturation with linear least-squares regression. ${ }^{23}$ The system, software, and analysis techniques have previously been validated on liquid phantoms with an accuracy of approximately $1 \%$ and in vivo. ${ }^{23}$

2-D fluorescence imaging was conducted in the epiillumination geometry with a $100-\mathrm{W}$ mercury lamp. NADH images were collected with a 360-nm bandpass excitation filter (40-nm bandwidth) and a 390-nm longpass dichroic beamsplitter. FAD images were collected with a $470-\mathrm{nm}$ bandpass excitation filter (40-nm bandwidth) and a 510-nm longpass dichroic beamsplitter. Fluorescence emission was collected with the same LCTF and charge-coupled device (CCD) described before for the hyperspectral arm. The LCTF was set to 480-nm and 530-nm emission for NADH and FAD imaging, respectively. Redox images were calculated by dividing the FAD image point by point by the NADH image. $^{30,34}$ To account for interanimal variability in the timecourse experiments, the redox ratio for each animal was normalized the first time point of the first region of interest within each animal. Note that the redox image represents the redox ratio of a superficial ( $\sim 200$ to $300 \mu \mathrm{m}$ thick) tissue layer probed by the excitation-emission wavelengths, ${ }^{35}$ and the hemoglobin saturation images show vessels from a simi- 
larly superficial tissue layer. ${ }^{22,23}$ OCT volumes indicate that this layer contains most of the blood vessels in the field of view. The spatial resolution of hyperspectral and redox imaging is defined by the NA of the objective $(5 \mu \mathrm{m})$.

The spectral domain OCT arm was illuminated by a Ti:sapphire laser source (Femtolasers, Vienna, Austria) centered at $790 \mathrm{~nm}$ with a $90-\mathrm{nm}$ full width at half-maximum. The OCT interferometer was common path, utilizing the top surface of the window chamber cover glass as the reference reflection, and the spectral interferogram was detected using a custom-made spectrometer (spectral resolution and coverage 0.08 and $164 \mathrm{~nm}$, respectively) with a 2048 element line scan CCD camera (Atmel, Aviiva, San Jose, California). The OCT system was driven by software that controls the lateral scanner and performs data acquisition and archiving (Bioptigen, Incorporated, Research Triangle park, North Carolina). 3-D blood flow images were collected with Doppler OCT, which measures phase changes due to flowing erythrocytes. ${ }^{24}$ Phase changes were calculated from multiple A-scans collected at the same position in the volume, and were related to flow velocity profiles by measuring the angle of incidence in the $3-\mathrm{D}$ volume. This method has previously been validated in flow phantoms. ${ }^{36}$ Doppler OCT volumes were collected over a $2 \times 1.5 \mathrm{~mm}$ area with $250 \times 125$ pixels (with a resulting resolution of 8 and $12 \mu \mathrm{m}$ in the lateral dimensions), and from $2 \mathrm{~mm}$ in the depth dimension with 1024 pixels, with a 1-ms integration time for each A-line. Ten repeated A-scans at each location were collected at each $x-y$ position for Doppler shift estimation. Cross sectional vessel flow profiles were fit to a second-order polynomial and corrected for the angle of incidence to provide velocity in millimeters per second. Maximum blood velocity $\left(V_{\max }\right)$ was determined from the peak of this fit, vessel diameter $(D)$ was determined from the zerocrossings of the fit, and the shear rate on the vessel wall was determined from the derivative of the fit $\left(d v_{z} / d r\right)$ evaluated at the vessel wall, assuming a Newtonian fluid. ${ }^{29}$ Note that in vessels with diameters 30 to $60 \mu \mathrm{m}$, the velocity profile is expected to be blunter than a parabola (this reflects the noncontinuum behavior of blood rather than non-Newtonian behavior). ${ }^{25,37-39}$ Thus, the estimated wall shear rate may be an underestimate in these calculations. However, our fit results suggest that the departure from a parabola is small, so the second-order polynomial fit is a reasonable approximation. The flow in the vessel is the average velocity in the vessel times the cross sectional area of the vessel $\left[V_{\max } / 2^{*} \pi^{*}(D / 2)^{2}\right]$.

\subsection{In Vivo Imaging}

All in vivo experiments were conducted under a protocol approved by the Duke University Institutional Animal Care and Use Committee. A titanium window chamber was surgically implanted under anesthesia (ketamine $100 \mathrm{mg} / \mathrm{kg}$ and xylazine $10 \mathrm{mg} / \mathrm{kg}$ IP) on the back of athymic nude mice (nu/nu, NCI, Frederick, Maryland). A window chamber tumor was established during chamber implantation by injecting $20 \mu \mathrm{L}$ of a single cell suspension $\left(8 \times 10^{6}\right.$ cells) of $\mathrm{FaDu}$ (human squamous cell carcinoma) cells into the dorsal skin fold prior to placing a 12 -mm-diam number 2 round glass coverslip (Erie Scientific, Portsmouth, New Hampshire) over the exposed tissue. Animals were housed in an on-site housing fa- cility with ad libitum access to food and water and standard 12-h light/dark cycles.

Redox and hemoglobin saturation imaging were validated on mice with a tumor-free window chamber. Perturbation experiments were performed with the mice under ketamine/ xylazine anesthesia (maintained at body temperature) while breathing air, then $100 \%$ oxygen through a nose cone, and then $100 \%$ nitrogen through a nose cone at $1-\mathrm{L} / \mathrm{min}$ flow rate.

For longitudinal imaging of tumor growth, animals were anesthetized with Isoflurane (1.5\% with oxygen) and maintained at body temperature. Imaging began when tumors reached 1 to $2 \mathrm{~mm}$ in diameter. A Doppler OCT volume (5-min acquisition), followed by hemoglobin saturation (30-s acquisition) and redox (15-sec acquisition) imaging were collected every $6 \mathrm{~h}$ for $36 \mathrm{~h}$. Vessel morphology maps were used to achieve spatial registration between each imaging session. Transition between microscope modes was approximately $1 \mathrm{~min}$. Mice were allowed to recover after each imaging session.

Note that no corrections were made to the data to account for the cardiac cycle. All vessels of interest were microvessels (diameter $<70 \mu \mathrm{m}$ ) that do not exhibit large changes in flow due to the cardiac cycle. Previous studies have used Fourier analysis of blood flow and $p \mathrm{O}_{2}$ in rats to show low-frequency fluctuations $(<2$ cycles $/ \mathrm{min})$ in both tumor and muscle tissues, which is much lower than the measured respiration rate and heart rate $(\sim 50$ to 60 and 300 to 400 cycles $/ \mathrm{min}$, respectively. $^{7}$

\subsection{Statistical Analysis}

Doppler OCT and hyperspectral data were collected over the 36-h time course from a total of five mice, and redox images were also collected over the time course from three of these mice. Three to six regions of interest were chosen within each tumor based on visual inspection of the hemoglobin saturation time course images. Vessel regions that demonstrated fluctuations in hemoglobin saturation over the time course, and that were spatially distributed across the surface of the tumor, were chosen as regions of interest. The mean and standard deviation of the hemoglobin saturation were measured at these vessel regions of interest, over an area within the inner diameter of the vessel and a slab thickness of no more than $20-\mu \mathrm{m}$. The Doppler velocity profile was measured at the same vessel cross section of interest. The mean and standard deviation of the redox ratio was measured from a 25 to $60-\mu \mathrm{m}$-diam circular area adjacent to the vessel. The redox area of interest was chosen to exclude blood vessels and to remain less than $100 \mu \mathrm{m}$ in diameter (the length of oxygen diffusion in tissue. ${ }^{40}$ A total of 21 regions of interest were analyzed over the time course, with 14 regions of interest that include redox ratio measurements. One of the animals for which redox images were collected included only six time points for the four regions of interest within that animal, due to tumor hemorrhage at the last time point.

Pearson's correlation coefficients were used to determine linear correlations between blood oxygenation (hemoglobin saturation), blood flow (maximum velocity, flow, vessel inner diameter, and shear rate), and metabolic demand (redox ratio). Significant correlations $(p<0.05)$ are reported for the pooled 
data (all regions of interest and time points grouped together), for which there were $n=143$ samples for the blood oxygenation and blood flow comparisons, and $n=94$ samples for the metabolic demand comparisons. As described before, the redox ratio was normalized to the first time point of the first region of interest within each animal. Thus, blood oxygenation and blood flow biomarkers were also normalized to the first time point of the first region of interest within each animal for comparisons with the redox ratio only.

Next, a linear mixed model was used to analyze repeated measures. This approach separates the interanimal variation from the total variation (thus increasing statistical power) to determine whether the biomarkers (i.e., hemoglobin saturation, redox ratio, maximum velocity, vessel inner diameter, flow, and shear rate) change over time. Let $Y_{i j k}$ denote the value of a biomarker from the $i$ 'th animal in the $j$ 'th region of interest (ROI) at the $k$ 'th time point. Assume $Y_{i j k}$ follows the linear mixed model $Y_{i j k}=\mu+t_{k}+b_{i j}+\varepsilon_{i j k}$ (model 1); where $\mu+t_{k}$ is the time effect, $b_{i j}$ is the animal specific random effect, and $\varepsilon_{i j k}$ are intra-animal errors. The time effect was tested by the F-statistic. A second mixed model was used to address the association of two variables adjusting for the covariate time. Model $2\left(Y_{i j k}=\mu+X_{i j k}+t_{k}+b_{i j}+\varepsilon_{i j k}\right)$ includes the covariate $X$, and the linear association of biomarker $X$ on the behavior of another biomarker $Y$ was tested with the F-statistic. Associations were tested between blood oxygenation (hemoglobin saturation), blood flow (maximum velocity, flow, vessel inner diameter, and shear rate), and metabolic demand (redox ratio) biomarkers.

\section{Results}

The results of perturbation experiments in a normal mouse window chamber (no tumor cells were injected) are shown in Fig. 1. To segment the vessels from background, the hemoglobin saturation images were thresholded by the total absorption image. The same mask was negatively applied to the redox images. Note that there are two distinct peaks in the hemoglobin saturation histogram for air and oxygen breathing [Fig. 1(b)], due to arteriolar and venous blood oxygenation. Nitrogen and oxygen breathing resulted in a decrease and increase, respectively, in both the redox ratio and the hemoglobin saturation compared to air breathing, as expected. The results in Fig. 1 were confirmed in a second mouse, and are consistent with previous reports. ${ }^{23,33}$ These perturbation experiments indicate that NADH and FAD signals have been properly isolated for redox imaging.

The hemoglobin saturation [Fig. 1(a)] and redox [Fig. 1(b)] images collected from one mouse over the 36-h time course are shown in Fig. 2. Longitudinal oxygen gradients are apparent in the hemoglobin saturation images, with decreased hemoglobin saturation levels within the tumor [Fig. 2(a), at the 1] compared with the tumor periphery [Fig. 2(a), at the 2]. These longitudinal gradients have been previously observed in studies employing phosphorescence lifetime imaging of $\mathrm{pO}_{2}{ }^{41,42}$ The redox images indicate that the redox ratio within the tumor is higher in areas with large-diameter oxygenated vessels [Fig. 2(b), at the 3]. Both the hemoglobin saturation and the redox ratio appear to vary with time.

Figure 3 shows an en-face view of the 3-D Doppler OCT volume (manually segmented in Amira software, Mercury

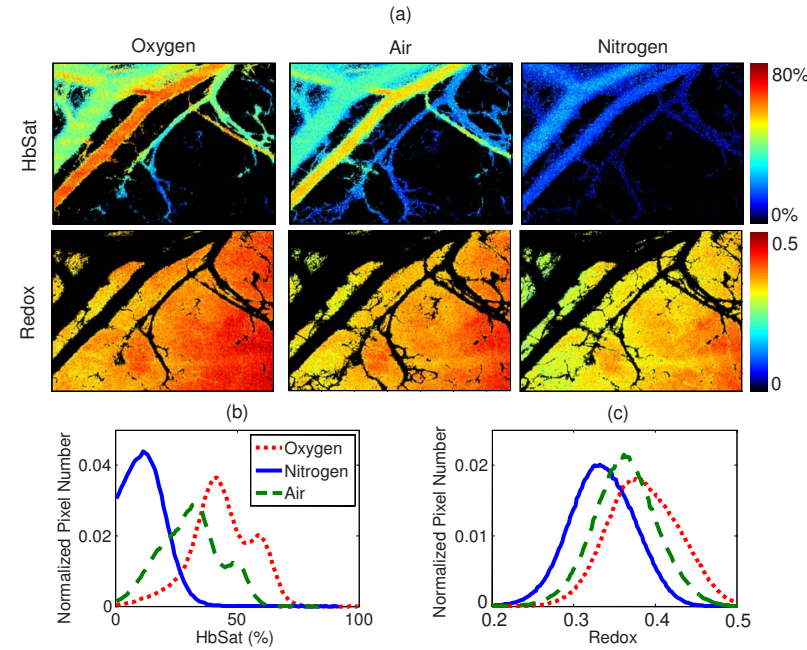

Fig. 1 Hemoglobin saturation (HbSat) and redox images taken from a nontumor-bearing window chamber of a mouse breathing oxygen, air, and nitrogen (a) at $1 \mathrm{~L} / \mathrm{min}$ through a nose cone. To segment the vessels from background, the hemoglobin saturation images were thresholded by the total absorption image. The same mask was negatively applied to the redox images. Histograms of (b) the hemoglobin saturation and (c) redox images show that the hemoglobin saturation and redox ratio increase with oxygen breathing and decrease with nitrogen breathing, as expected. Image sizes are $2 \times 1.5 \mathrm{~mm}$.

Systems, Chelmsford, Massachusetts) from the zero-hour time point [Fig. 3(a)] of the same animal shown in Fig. 2. Plots of the cross sectional vessel velocity profile from one vessel [arrow, Fig. 3(a)] are shown along with the second-order polynomial fit and the R-squared value of the fit for each time point [Figs. 3(b)-3(h)]. The shape and peak of the velocity profile changes with time, indicating changes in vessel diameter, shear rate, and maximum vessel velocity over the 36-h time course.

Figure 4 shows quantitative measures of the redox ratio [Fig. 4(b)], hemoglobin saturation [Fig. 4(c)], vessel maximum velocity [Fig. 4(d)], vessel inner diameter [Fig. 4(e)], flow [Fig. 4(f)], and shear rate [Fig. 4(g)] over the 36-h time course in six regions of interest [Fig. 4(a)] from the same animal shown in Figs. 2 and 3. These plots indicate that all variables change with time within the same region of interest within the same animal. All regions of interest appear to vary together with time for the redox ratio [Fig. 4(b)] and for the hemoglobin saturation [Fig. 4(c)]. However, each region of interest appears to vary differently with time compared with the other five regions of interest for the blood flow parameters [Figs. 4(d)-4(g)].

The spatial distribution of the redox ratio with respect to the nearest vessel is shown in Fig. 5 for tumors and normal tissues. There is a significant $(p<0.05)$ decrease in the redox ratio for points greater than $30 \mu \mathrm{m}$ from the nearest vessel, compared to the point closest to the nearest vessel (point zero). This is in agreement with previous reports that measured $\mathrm{pO}_{2}$ as a function of distance from the nearest vessel. ${ }^{15,16}$ However, the normal tissues showed no significant increase or decrease in the redox ratio as a function of distance from the nearest vessel. 
(a)

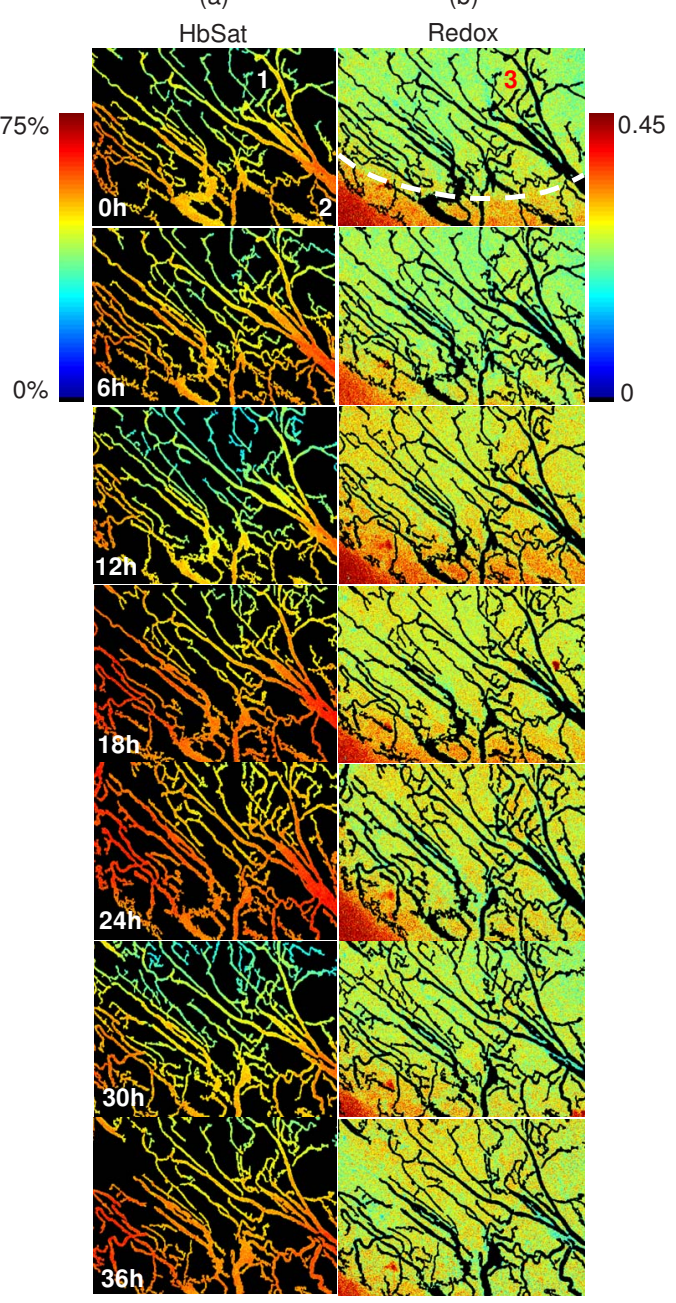

Fig. 2 (a) Hemoglobin saturation and (b) redox images taken from one mouse over the 36-h time course. The time of imaging in hours is indicated in the lower left corner of each hemoglobin saturation image. For the purposes of this figure only, the hemoglobin saturation images were thresholded by the total absorption image, and then by a 15- $\mu$ m-diam circular kernel. The kernel excluded pixels with an absorption value smaller than the average of the $15-\mu \mathrm{m}$-diam kernel. The mask was applied to hemoglobin saturation images to isolate vessels from the nonvascular background, and the same vessel mask was negatively applied to the redox images. The tumor boundary is indicated by a white dashed line in (b). Image sizes are $2 \times 1.5 \mathrm{~mm}$.

The relationship between the biomarkers for blood oxygenation (hemoglobin saturation), blood flow (maximum velocity, flow, vessel inner diameter, and shear rate), and metabolic demand (redox ratio) were tested with the Pearson's correlation coefficient (Table 1) for the pooled data (all regions of interest and time points from all animals grouped together). The correlation coefficients $(r)$ in Table 1 are shown for biomarker relationships for which a significant $(p$ $<0.05)$ linear correlation was found. The hemoglobin saturation is positively correlated with the maximum velocity, flow, and shear rate (HbSat line of Table 1$)$, and the redox ratio is positively correlated with the hemoglobin saturation, maximum velocity, flow, and diameter. All statistically significant correlations are weak $(r \leqslant 0.5)$, which indicates that many
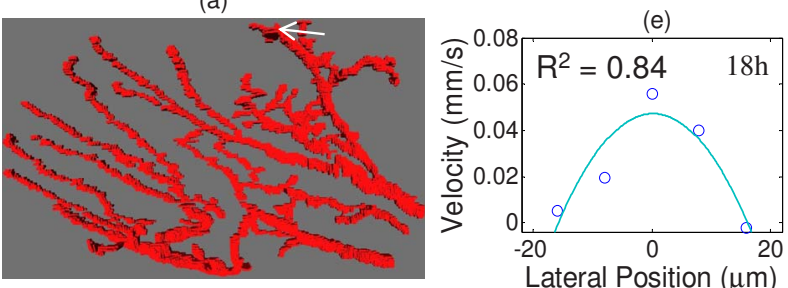

(b)

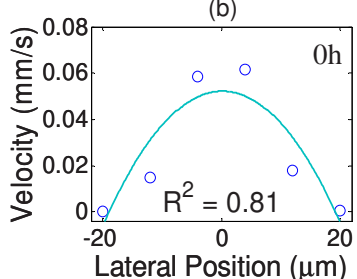

(c)

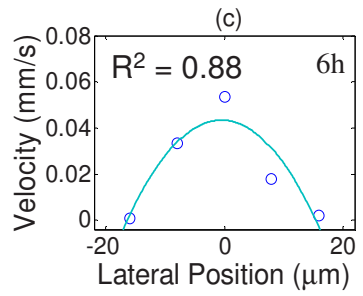

(d)

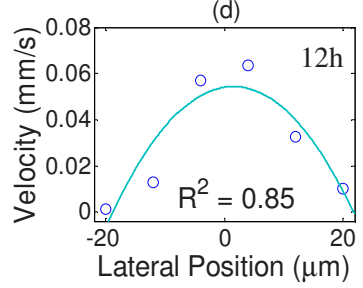

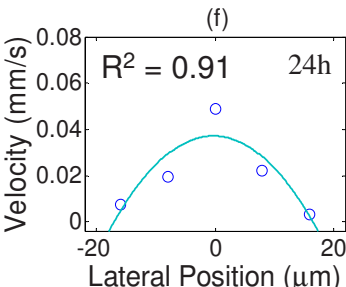

(g)

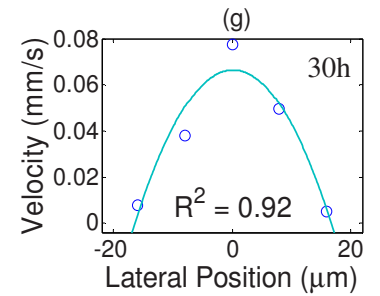

(h)

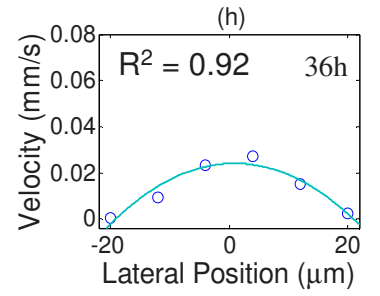

Fig. 3 An en-face view of the 3-D Doppler OCT volume (manually segmented in Amira software, Mercury Systems) from (a) the zerohour time point of the same animal shown in Fig. $2(2 \times 1.5-\mathrm{mm}$ imaged area, $0.6 \mathrm{~mm}$ depth). Plots of the cross sectional vessel velocity profile from one vessel [arrow in (a)] are shown along with the second-order polynomial fit and the R-squared value of the fit for each time point (b) through (h). The time of imaging in hours is indicated in the upper right corner of each plot.

factors contribute to the variability of each biomarker, as expected. ${ }^{11}$ The linear mixed model determined that all biomarkers significantly change $(p<0.05)$ with time (Fig. 6), and that the vessel diameter is associated with the redox ratio of the adjacent tissue $(p<0.05)$ after adjusting for the effect of time. No other biomarker pairs were found to have significant associations $(p>0.05)$ when adjusting for the effect of time.

\section{Discussion}

Multifunctional optical imaging of oxygen supply and demand has several advantages over traditional techniques. The combination of OCT, absorption, and fluorescence imaging provides quantitative information on blood flow, blood oxygenation, and metabolic demand, respectively, in living tissues. This combination of optical imaging modalities is noninvasive, relatively inexpensive, and fast. These attributes are well suited for long-term repeated measures in vivo. We have demonstrated the attributes of this imaging platform for the study of cycling hypoxia in tumors in the dorsal skin fold mouse window chamber. This is the first demonstration of 

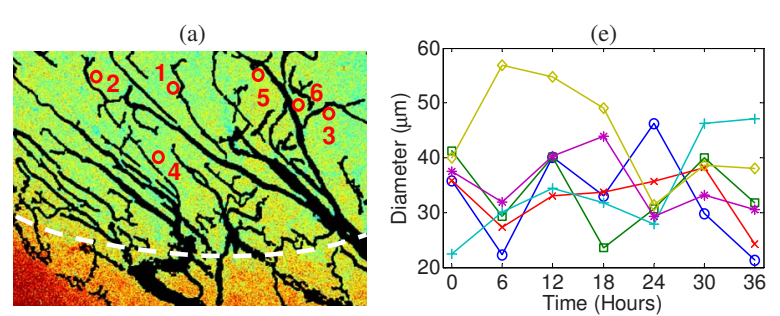

(b)
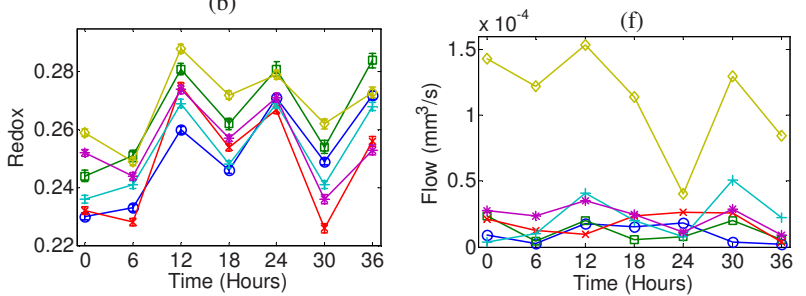

(c)

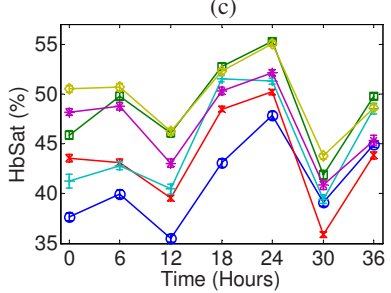

(d)
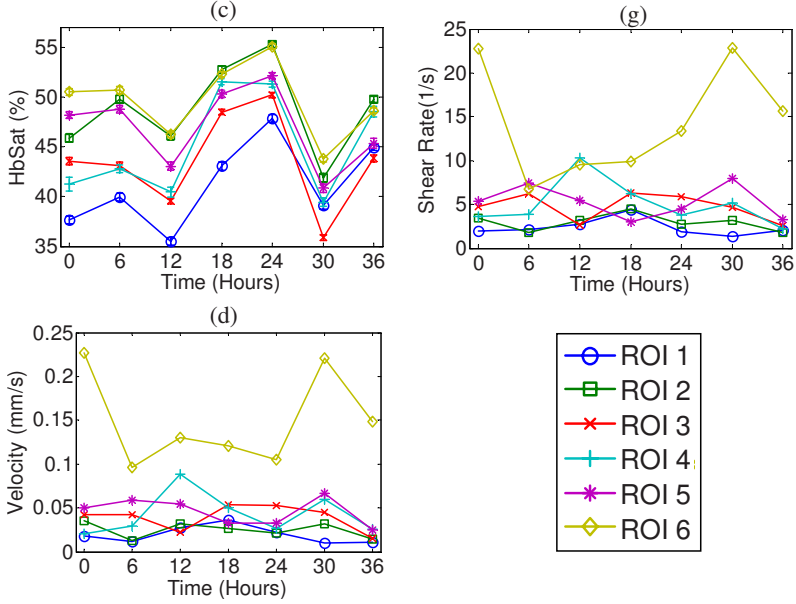

Fig. 4 Quantitative measures of (b) the redox ratio, (c) hemoglobin saturation, (d) vessel maximum velocity, (e) vessel inner diameter, (f) flow, and (g) shear rate over the 36-h time course in (a) six regions of interest from the same animal shown in Figs. 2 and 3. The mean and standard error are plotted for (b) the redox ratio and (c) hemoglobin saturation, and the mean (d) through ( $g$ ) is plotted for the remaining variables.

combined noninvasive monitoring of oxygen supply and metabolic demand in tumors in vivo.

All biomarkers (hemoglobin saturation, redox ratio, maximum velocity, vessel inner diameter, flow, and shear rate) significantly changed with time $(p<0.05)$ over the $36-\mathrm{h}$ imaging period, which supports previous reports that blood oxygenation, blood flow, and metabolic demand all contribute to cycling hypoxia in tumors. ${ }^{3}$ These variations in oxygen supply and demand are superimposed on a decline in metabolic demand with distance from the nearest vessel in tumors (Fig. 5 ), in agreement with previous interstitial $p \mathrm{O}_{2}$ microelectrode measurements. ${ }^{15,16}$ Our results indicate a positive correlation between the blood flow, blood oxygenation, and metabolic demand in tumors (Table 1), but the correlation between these biomarkers is weak and suggests that no single biomarker can fully explain the behavior of another. ${ }^{11}$ When adjusting for the effect of time, the vessel diameter was found to be associated with the redox ratio of the adjacent tissue, which indicates that vessel diameter plays a key role in tumor metabolic activity and cycling hypoxia in tumors. ${ }^{43}$ Future studies will exploit these biomarkers to investigate functional changes in

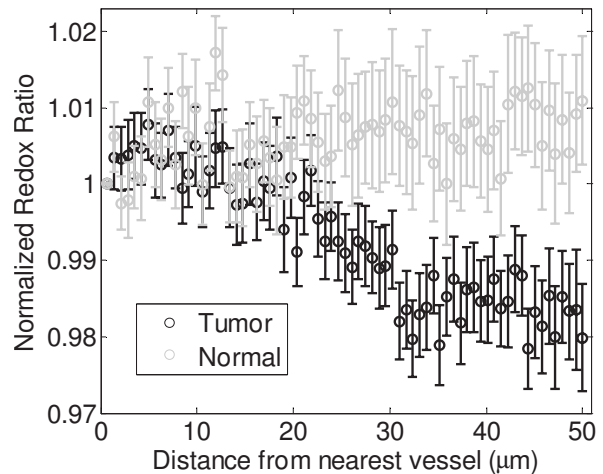

Fig. 5 Normalized redox ratio as a function of distance from the nearest vessel for tumors (compiled from $n=94$ line profiles corresponding to the 94 regions of interest from the three redox animals across the entire tumor time course), and for normal tissues (compiled from $n$ $=36$ line profiles from six regions of interest from each of six normal animals). All profiles were normalized to the point closest to the vessel before averaging across all line profiles. For the tumors, there is a significant $(p<0.05)$ decrease in the redox ratio between the point closest to the vessel (point zero) and all points greater than $30 \mu \mathrm{m}$ from point zero. Normal tissues did not show a significant increase or decrease in the redox ratio as a function of the distance from the nearest vessel. Error bars are standard error.

oxygen supply and demand in response to therapeutic strategies. For example, it is possible that antiangiogenic therapies may stabilize cycling hypoxia by reducing the magnitude of changes in vascular diameter and blood flow rate over time. ${ }^{3}$ It is anticipated, however, that antiangiogenic therapies would have less influence on redox ratio. The combination of technologies presented here would permit a direct test of this hypothesis.

Extracted biomarkers including the hemoglobin saturation, ${ }^{44,45}$ maximum velocity and vessel diameter, ${ }^{14}$ and shear rate, ${ }^{46}$ are all in qualitative agreement with previous studies that employed different techniques in window chamber tumors, as well as a previous study that employed the same techniques in a different window chamber tumor model. ${ }^{28}$ Variations in red blood cell flux and $\mathrm{pO}_{2},{ }^{15}$ hemoglobin oxygen saturation, ${ }^{23}$ and diameter ${ }^{44}$ have previously

Table 1 Pearson correlation coefficients for hemoglobin saturation (HbSat) versus maximum velocity $\left(V_{\max }\right)$, flow, diameter, and shear rate (top line) and for the normalized redox ratio, normalized hemoglobin saturation, normalized maximum velocity, normalized flow, normalized diameter and normalized shear rate (bottom line). The data are compiled from all regions of interest and time points grouped together, with $n=143$ observations for the top line and $n=94$ observations for the bottom line. The correlation coefficient is listed for comparisons with $p<0.05$.

\begin{tabular}{lccccc}
\hline & $\begin{array}{c}V_{\text {max }} \\
(\mathrm{mm} / \mathrm{s})\end{array}$ & $\begin{array}{c}\text { Flow } \\
\left(\mathrm{mm}^{3} / \mathrm{s}\right)\end{array}$ & $\begin{array}{c}\text { Diameter } \\
(\mu \mathrm{m})\end{array}$ & $\begin{array}{c}\text { Shear rate } \\
(1 / \mathrm{s})\end{array}$ \\
\hline HbSat $(\%)$ & 0.49 & 0.37 & $p>0.05$ & 0.49 \\
& HbSat & $V_{\text {max }}$ & Flow & Diameter & Shear rate \\
Redox & 0.50 & 0.28 & 0.32 & 0.43 & $p>0.05$ \\
\hline
\end{tabular}




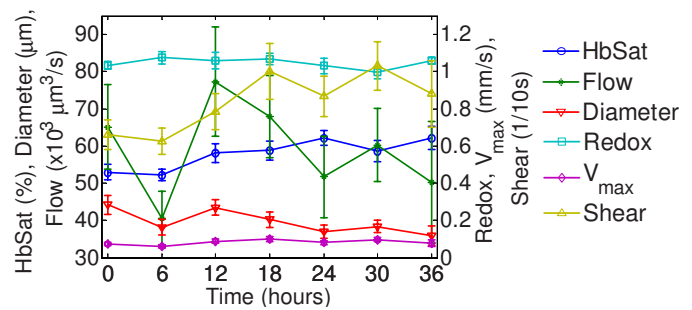

Fig. 6 Longitudinal variations in all biomarkers as a function of time. Values at each time point were averaged across all regions of interest and animals for the hemoglobin saturation (HbSat), flow, diameter, maximum velocity $\left(V_{\max }\right)$, and shear rate $(n=21)$, as well as for the redox ratio $(n=14)$. Note that the 36 -h time point has four fewer samples (see Sec. 2). The linear mixed model determined that all biomarkers significantly change $(p<0.05)$ with time, and that the vessel diameter is associated with the redox ratio of the adjacent tissue $(p<0.05)$ after adjusting for the effect of time. Error bars are standard error.

been observed in window chamber tumors over time scales ranging from one hour to several days.

Previous studies have employed different methods to investigate the relationship between some of the biomarkers presented in this work. Intravascular $p \mathrm{O}_{2}$ and blood flow rate, as well as interstitial $p \mathrm{O}_{2}$ and red blood cell velocity or vessel diameter, were found to have no correlation in a previous study. ${ }^{13}$ Helmlinger et al. used phosphorescence lifetime imaging to quantify $p \mathrm{O}_{2}$ and video microscopy to quantify red blood cell velocity and vessel diameter. Although hemoglobin oxygen saturation and $p \mathrm{O}_{2}$ are related, they are not the same endpoint, and video microscopy provides a 2-D projection rather than an absolute measure of blood velocity. The study design and tumor models employed in the previous and current studies also differ. Previous studies employing the same techniques as the current study have found a significant correlation between the hemoglobin saturation and blood velocity, blood flow, and shear rate in a different tumor model. ${ }^{28}$ Additional studies ${ }^{15}$ indicate that vessel remodeling with tumor growth alters flow distributions, and the relationship between blood flow and tissue oxygenation in tumors is heterogeneous.

Demand for oxygen in tumor cells is created by the normal respirative process of the cells. ${ }^{32}$ The redox ratio is a measure of cell respiration (oxygen demand) ${ }^{30}$ while the hemoglobin oxygen saturation is one component of oxygen supply. Therefore, it is intuitive that the hemoglobin saturation of a vessel is somewhat related to the redox ratio of the surrounding tissue. However, we do not expect a one-to-one correlation between the redox ratio and hemoglobin saturation. Oxygen delivery is affected by the hemoglobin saturation as well as the vascular architecture, hematocrit, and blood flow. ${ }^{32}$ The redox ratio is indicative of changes in oxygen supply as well as metabolite availability and mitochondrial membrane potential. ${ }^{47}$ Thus, the redox ratio, blood flow and hemoglobin saturation provide complementary information.

Figure 5 shows a decrease in the redox ratio with increasing distance from the nearest vessel in tumors, and no such decrease in the redox ratio with distance from the nearest vessel in normal tissues. We hypothesize that this tumor gradient is due to increased metabolic activity coupled with com- promised microvascular oxygen transport in tumors compared to normal tissues. Future studies will investigate this hypothesis.

In this study, absorption and fluorescence microscopy were combined with OCT to unravel the contributions of blood flow, blood oxygenation, and metabolic demand on cycling hypoxia. These findings could guide the design and development of therapeutic strategies to mitigate cycling hypoxia, and thus reduce tumor resistance to radiation and chemotherapies. Second, this study experimentally demonstrates the attributes of our multifunctional optical imaging approach for mapping oxygen supply and demand in vivo. This approach has broad applicability in the study of other diseases that are affected by oxygen supply and demand, such as cerebral hypoxia (stroke), cardiac ischemia, and Alzheimer's disease. Moreover, these processes can be studied with multifunctional optical imaging in vivo and do not require contrast-enhancing dyes.

\section{Acknowledgments}

The authors would like to thank Greg Palmer and Hansford Hendargo for their technical expertise. This research was supported by NIH/NCI CA40355, NIH EB006338, and NIH 014743. Author Skala acknowledges fellowship support through the NIH/NCI (F32 CA130309). Joseph Izatt has a financial interest in Bioptigen, Inc. as Chief Technology Officer.

\section{References}

1. J. M. Brown and W. R. Wilson, "Exploiting tumour hypoxia in cancer treatment," Nature Rev. 4(6), 437-447 (2004).

2. P. Vaupel and A. Mayer, "Hypoxia in cancer: significance and impact on clinical outcome," Cancer Metastasis Rev. 26(2), 225-239 (2007).

3. M. W. Dewhirst, Y. Cao, and B. Moeller, "Cycling hypoxia and free radicals regulate angiogenesis and radiotherapy response," Nature Rev. 8(6), 425-437 (2008).

4. M. W. Dewhirst, "Intermittent hypoxia furthers the rationale for hypoxia-inducible factor-1 targeting," Cancer Res. 67(3), 854-855 (2007).

5. L. I. Cardenas-Navia, D. Yu, R. D. Braun, D. M. Brizel, T. W. Secomb, and M. W. Dewhirst, "Tumor-dependent kinetics of partial pressure of oxygen fluctuations during air and oxygen breathing," Cancer Res. 64(17), 6010-6017 (2004).

6. K. G. Brurberg, H. K. Skogmo, B. A. Graff, D. R. Olsen, and E. K. Rofstad, "Fluctuations in pO2 in poorly and well-oxygenated spontaneous canine tumors before and during fractionated radiation therapy," Radiol. Oncol. 77(2), 220-226 (2005).

7. R. D. Braun, J. L. Lanzen, and M. W. Dewhirst, "Fourier analysis of fluctuations of oxygen tension and blood flow in R3230Ac tumors and muscle in rats," Am. J. Physiol. 277(2 Pt 2), H551-568 (1999).

8. K. G. Brurberg, B. A. Graff, and E. K. Rofstad, "Temporal heterogeneity in oxygen tension in human melanoma xenografts," $\mathrm{Br}$. J. Cancer 89(2), 350-356 (2003).

9. G. L. Semenza, "Targeting HIF-1 for cancer therapy," Nature Rev. 3(10), 721-732 (2003).

10. H. Kimura, R. D. Braun, E. T. Ong, R. Hsu, T. W. Secomb, D. Papahadjopoulos, K. Hong, and M. W. Dewhirst, "Fluctuations in red cell flux in tumor microvessels can lead to transient hypoxia and reoxygenation in tumor parenchyma," Cancer Res. 56(23), 55225528 (1996)

11. T. W. Secomb, R. Hsu, E. T. Ong, J. F. Gross, and M. W. Dewhirst, "Analysis of the effects of oxygen supply and demand on hypoxic fraction in tumors," Acta Oncol. 34(3), 313-316 (1995).

12. K. O. Hicks, F. B. Pruijn, T. W. Secomb, M. P. Hay, R. Hsu, J. M. Brown, W. A. Denny, M. W. Dewhirst, and W. R. Wilson, "Use of three-dimensional tissue cultures to model extravascular transport and predict in vivo activity of hypoxia-targeted anticancer drugs," $J$. Natl. Cancer Inst. 98(16), 1118-1128 (2006).

13. G. Helmlinger, F. Yuan, M. Dellian, and R. K. Jain, "Interstitial pH 
and $\mathrm{pO} 2$ gradients in solid tumors in vivo: high-resolution measurements reveal a lack of correlation," Nat. Med. 3(2), 177-182 (1997).

14. M. W. Dewhirst, R. Oliver, C. Y. Tso, C. Gustafson, T. Secomb, and J. F. Gross, "Heterogeneity in tumor microvascular response to radiation," Int. J. Radiat. Oncol., Biol., Phys. 18(3), 559-568 (1990).

15. J. Lanzen, R. D. Braun, B. Klitzman, D. Brizel, T. W. Secomb, and M. W. Dewhirst, "Direct demonstration of instabilities in oxygen concentrations within the extravascular compartment of an experimental tumor," Cancer Res. 66(4), 2219-2223 (2006).

16. M. W. Dewhirst, T. W. Secomb, E. T. Ong, R. Hsu, and J. F. Gross, "Determination of local oxygen consumption rates in tumors," Cancer Res. 54(13), 3333-3336 (1994).

17. P. Vaupel, K. Ostheimer, and W. Muller-Klieser, "Circulatory and metabolic responses of malignant tumors during localized hyperthermia," J. Cancer Res. Clin. Oncol. 98(1), 15-29 (1980).

18. P. Vaupel, F. Kallinowski, and P. Okunieff, "Blood flow, oxygen and nutrient supply, and metabolic microenvironment of human tumors: a review," Cancer Res. 49(23), 6449-6465 (1989).

19. L. K. Shankar, J. M. Hoffman, S. Bacharach, M. M. Graham, J. Karp, A. A. Lammertsma, S. Larson, D. A. Mankoff, B. A. Siegel, A. Van den Abbeele, J. Yap, and D. Sullivan, "Consensus recommendations for the use of 18F-FDG PET as an indicator of therapeutic response in patients in National Cancer Institute Trials," J. Nucl. Med. 47(6), 1059-1066 (2006).

20. P. Som, H. L. Atkins, D. Bandoypadhyay, J. S. Fowler, R. R. MacGregor, K. Matsui, Z. H. Oster, D. F. Sacker, C. Y. Shiue, H Turner, C. N. Wan, A. P. Wolf, and S. V. Zabinski, "A fluorinated glucose analog, 2-fluoro-2-deoxy-D-glucose (F-18): nontoxic tracer for rapid tumor detection," J. Nucl. Med. 21(7), 670-675 (1980).

21. C. Dedeugd, M. Wankhede, and B. S. Sorg, "Multimodal optical imaging of microvessel network convective oxygen transport dynamics," Appl. Opt. 48(10), D187-197 (2009).

22. B. S. Sorg, M. E. Hardee, N. Agarwal, B. J. Moeller, and M. W. Dewhirst, "Spectral imaging facilitates visualization and measurements of unstable and abnormal microvascular oxygen transport in tumors," J. Biomed. Opt. 13(1), 014026 (2008).

23. B. S. Sorg, B. J. Moeller, O. Donovan, Y. Cao, and M. W. Dewhirst, "Hyperspectral imaging of hemoglobin saturation in tumor microvasculature and tumor hypoxia development," J. Biomed. Opt. 10(4), 44004 (2005).

24. J. A. Izatt, M. D. Kulkarni, S. Yazdanfar, J. K. Barton, and A. J. Welch, "In vivo bidirectional color Doppler flow imaging of picoliter blood volumes using optical coherence tomography," Opt. Lett. 22(18), 1439-1441 (1997).

25. J. Moger, S. J. Matcher, C. P. Winlove, and A. Shore, "Measuring red blood cell flow dynamics in a glass capillary using Doppler optical coherence tomography and Doppler amplitude optical coherence tomography," J. Biomed. Opt. 9(5), 982-994 (2004).

26. M. Khurana, E. H. Moriyama, A. Mariampillai, and B. C. Wilson, "Intravital high-resolution optical imaging of individual vessel response to photodynamic treatment," J. Biomed. Opt. 13(4), 040502 (2008).

27. V. X. Yang, S. J. Tang, M. L. Gordon, B. Qi, G. Gardiner, M. Cirocco, P. Kortan, G. B. Haber, G. Kandel, I. A. Vitkin, B. C. Wilson, and N. E. Marcon, "Endoscopic Doppler optical coherence tomography in the human GI tract: initial experience," Gastrointest. Endosc. 61(7), 879-890 (2005)

28. M. C. Skala, A. Fontanella, H. Hendargo, M. W. Dewhirst, and J. A. Izatt, "Combined hyperspectral and spectral domain optical coherence tomography microscope for noninvasive hemodynamic imaging," Opt. Lett. 34(3), 289-291 (2009).

29. T. G. van Leeuwen, M. D. Kulkarni, S. Yazdanfar, A. M. Rollins, and J. A. Izatt, "High-flow-velocity and shear-rate imaging by use of color Doppler optical coherence tomography," Opt. Lett. 24(22), 1584-1586 (1999).
30. B. Chance, B. Schoener, R. Oshino, F. Itshak, and Y. Nakase, "Oxidation-reduction ratio studies of mitochondria in freeze-trapped samples. NADH and flavoprotein fluorescence signals," J. Biol. Chem. 254(11), 4764-4771 (1979).

31. R. Drezek, C. Brookner, I. Pavlova, I. Boiko, A. Malpica, R. Lotan, M. Follen, and R. Richards-Kortum, "Autofluorescence microscopy of fresh cervical-tissue sections reveals alterations in tissue biochemistry with dysplasia," Photochem. Photobiol. 73(6), 636-641 (2001).

32. C. J. Gulledge and M. W. Dewhirst, "Tumor oxygenation: a matter of supply and demand," Anticancer Res. 16(2), 741-749 (1996).

33. N. Ramanujam, R. R. Kortum, S. Thomsen, A. M. Jansen, M. Follen, and B. Chance, "Low temperature fluorescence imaging of freezetrapped human cervical tissues," Opt. Express 8, 335-343 (2001).

34. M. C. Skala, K. M. Riching, A. Gendron-Fitzpatrick, J. Eickhoff, K. W. Eliceiri, J. G. White, and N. Ramanujam, "In vivo multiphoton microscopy of NADH and FAD redox states, fluorescence lifetimes, and cellular morphology in precancerous epithelia," Proc. Natl. Acad. Sci. U.S.A. 104(49), 19494-19499 (2007).

35. N. Ramanujam, "Fluorescence spectroscopy of neoplastic and nonneoplastic tissues," Neoplasia 2(1-2), 89-117 (2000).

36. A. Davis, J. Izatt, and F. Rothenberg, "Quantitative measurement of blood flow dynamics in embryonic vasculature using spectral Doppler velocimetry," Anat. Rec. 292(3), 311-319 (2009).

37. P. Bagchi, "Mesoscale simulation of blood flow in small vessels," Biophys. J. 92(6), 1858-1877 (2007).

38. J. J. Bishop, P. R. Nance, A. S. Popel, M. Intaglietta, and P. C. Johnson, "Effect of erythrocyte aggregation on velocity profiles in venules," Am. J. Physiol. 280(1), H222-236 (2001).

39. M. Sharan and A. S. Popel, "A two-phase model for flow of blood in narrow tubes with increased effective viscosity near the wall," Biorheology 38(5-6), 415-428 (2001).

40. K. Groebe and P. Vaupel, "Evaluation of oxygen diffusion distances in human breast cancer xenografts using tumor-specific in vivo data: role of various mechanisms in the development of tumor hypoxia," Int. J. Radiat. Oncol., Biol., Phys. 15(3), 691-697 (1988).

41. M. W. Dewhirst, E. T. Ong, R. D. Braun, B. Smith, B. Klitzman, S. M. Evans, and D. Wilson, "Quantification of longitudinal tissue pO2 gradients in window chamber tumours: impact on tumour hypoxia," Br. J. Cancer 79(11-12), 1717-1722 (1999).

42. K. Erickson, R. D. Braun, D. Yu, J. Lanzen, D. Wilson, D. M. Brizel, T. W. Secomb, J. E. Biaglow, and M. W. Dewhirst, "Effect of longitudinal oxygen gradients on effectiveness of manipulation of tumor oxygenation," Cancer Res. 63(15), 4705-4712 (2003).

43. A. R. Pries, A. J. M. Cornelissen, A. A. Sloot, M. Hinkeldey, M. R. Dreher, M. Höpfner, M. W. Dewhirst, and T. W. Secomb, "Structural adaptation and heterogeneity of normal and tumor microvascular networks," PLOS Comput. Biol. 5(5), e1000394 (2009).

44. M. W. Dewhirst, H. Kimura, S. W. Rehmus, R. D. Braun, D. Papahadjopoulos, K. Hong, and T. W. Secomb, "Microvascular studies on the origins of perfusion-limited hypoxia," Br. J. Cancer 27, S247251 (1996)

45. B. R. Duling and R. M. Berne, "Longitudinal gradients in periarteriolar oxygen tension. A possible mechanism for the participation of oxygen in local regulation of blood flow," Circ. Res. 27(5), 669-678 (1970).

46. Y. Tsuzuki, D. Fukumura, B. Oosthuyse, C. Koike, P. Carmeliet, and R. K. Jain, "Vascular endothelial growth factor (VEGF) modulation by targeting hypoxia-inducible factor-1alpha-- $>$ hypoxia response element--> VEGF cascade differentially regulates vascular response and growth rate in tumors," Cancer Res. 60(22), 6248-6252 (2000).

47. G. M. Palmer, R. J. Viola, T. Schroeder, P. S. Yarmolenko, M. W. Dewhirst, and N. Ramanujam, "Quantitative diffuse reflectance and fluorescence spectroscopy: tool to monitor tumor physiology in vivo," J. Biomed. Opt. 14(2), 024010 (2009). 